Karl-Franzens Universität Graz

Technische Universität Graz

Medizinische Universität Graz

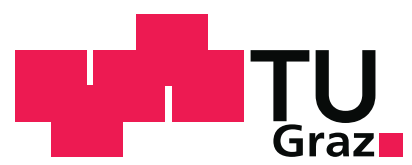

\title{
PDE constrained optimization of electrical defibrillation in a 3D ventricular slice geometry.
}
C. Nagaiah
K. Kunisch
G. Plank

SFB-Report No. 2015-019

September 2015

This article is a preprint. It was published in International Journal for Numerical Methods in Biomedical Engineering by Wiley. The version of record is available at http://dx.doi.org/doi:10.1002/cnm. 2742.

Supported by the Austrian Science Fund (FWF)

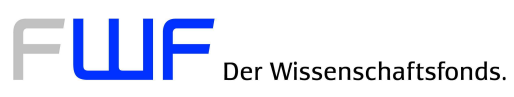

SFB sponsors:

- Austrian Science Fund (FWF)

- University of Graz

- Graz University of Technology

- Medical University of Graz

- Government of Styria

- City of Graz

Das Land

Steiermark 


\title{
PDE constrained optimization of electrical defibrillation in a 3D ventricular slice geometry
}

\author{
Nagaiah Chamakuri ${ }^{1 *}$, Karl Kunisch ${ }^{1,2 \dagger}$, Gernot Plank ${ }^{3 \ddagger}$ \\ ${ }^{1}$ Radon Institute for Computational and Applied Mathematics, \\ Altenbergerstr. 69, Linz, A-4040 Austria. \\ 2 Institute of Mathematics and Scientific Computing, \\ University of Graz, Heinrichstr. 36, Graz, A-8010 Austria. \\ ${ }^{3}$ Institute of Biophysics, Medical University of Graz, \\ Harrachgasse 21, Graz, A-8010 Austria.
}

July 4, 2016

\begin{abstract}
A computational study of an optimal control approach for cardiac defibrillation in a $3 \mathrm{D}$ geometry is presented. The cardiac bioelectric activity at the tissue and bath volumes is modeled by the bidomain model equations. The model includes intramural fiber rotation, axially symmetric around the fiber direction, and anisotropic conductivity coefficients which are extracted from a histological image. The dynamics of the ionic currents are based on the regularized Mitchell-Schaeffer model. The controls enter in the form of electrodes which are placed at the boundary of the bath volume with the goal of dampening undesired arrhythmias. The numerical optimization is based on Newton techniques. We demonstrated the parallel architecture environment for the computation of potentials on multidomains and for the higher order optimization techniques.
\end{abstract}

Keywords: electrophysiology, regularized Mitchell-Schaeffer model, cardiac arrhythmia, bidomain model, PDE constraint optimization, finite element method, second order optimization methods.

\section{Introduction}

Cardiac fibrillation is the breakdown of the organized electrical activity in the heart into disorganized self-sustained electrical activation patterns. Such fibrillatory episodes when affecting the ventricles, i.e. the main pumping chambers of the heart, result in loss of cardiac output and, unless timely intervention is administered,

\footnotetext{
*nagaiah.chamakuri@ricam. oeaw.ac.at

$\dagger$ karl.kunisch@uni-graz.at

${ }^{\ddagger}$ gernot.plank@medunigraz.at
} 
death quickly ensues. The only known effective therapy for lethal disturbances in cardiac rhythm is defibrillation, the delivery of a strong electric shock to the heart. This technique, as accomplished nowadays by automatic, implantable cardioverterdefibrillator (ICD) devices, constitutes the most effective means of combating sudden cardiac death [2]. Several multi-center clinical trials have provided consistent evidence that ICD therapy prolongs patient life.

Despite undeniable survival benefits of ICD implant [3], there are several known adverse effects secondary to the administration of strong electrical shocks which motivate experimental [22] and theoretical research $[19,26]$ to further improve safety and efficacy of the therapy. For instance, tissue in the proximity of shock electrodes may be permanently damaged by electroporation [17, 35], which is an inevitable consequence of the high field strength required to achieve defibrillation success. Even more importantly, psychological effects on patients play a non-negligible role. Conscious patients may perceive shock delivery as extremely painful which leads to traumatization and reduction in quality of life [11].

It is well established that success and failure of defibrillation shocks as well as their adverse side effects depend on the waveform of shock delivery. For instance, defibrillation is achieved with biphasic shocks more easily than with monophasic shocks, requiring significantly less energy [43] or that shocks are more effective with truncated waveforms [36]. With increasing clinical relevance of defibrillation therapy finding of optimal shock waveforms has become an important research topic. The two main design objectives in waveform design are to minimize delivered energy as well as minimize peak voltage and current. Besides empirical studies simplified $\mathrm{RC}$ network models have been used for waveform optimization [20]. While the RC model as a first order approximation neglects several important factors such as the non-linearity of cell membranes, the spatial distribution of the applied field or tissue heterogeneity [38], all major predictions have been verified in experimental studies.

A more comprehensive approach which appreciates all known biophysical mechanisms involved in the defibrillation process employs the cardiac bidomain equations [39], a homogenized continuum representation of electrical activity in cardiac tissue. In bidomain theory it is assumed that cardiac tissue consists of two spaces, an intracellular space and an extracellular or interstitial space, which are interpenetrating, but separated by a cellular membrane at any point in space. Computational bidomain modeling has proven to be an extremely powerful tool for gaining better insight into the biophysical mechanisms [37, 38] which govern success and failure of defibrillation shocks. More recently, PDE constrained optimization techniques have been applied, for the first time, to the bidomain model to predict optimized shock waveforms $[15,16,14]$. In these preceding studies, applicability of optimal control was investigated using model representations of the heart which were simplified in several regards. Cardiac anatomy and structure was approximated as a $2 \mathrm{D}$ sheet with spatially non-varying fiber orientation, but with random variations in tissue conductivity, and cellular dynamics was modeled using the phenomenological Fitzhugh-Nagumo model [16, 14]. Moreover, it was assumed that the heart is surrounded by a non-conductive material which is a significant limitation when studying defibrillation. Not only are bath loading effects $[7,8]$ ignored, also shock electrodes cannot be placed in the surrounding bath, as this is the case in any experimental or clinical setting. In the current study some of these previously assumed simplifications have been lifted. In particular, an anatomically realistic rabbit biventricular geometry with experimentally based fiber and sheet orientations, immersed 
in a conductive bath medium, is considered and cellular dynamics is represented by the more recent Mitchell-Schaeffer model.

The optimal control approach to cardiac defibrillation is to determine an applied external stimulus in such a way that it optimizes a given design objective, which is, in our case, the restoration of a tissue state in which fibrillatory propagation cannot be maintained. This can be achieved by driving the whole tissue to a resting state, or equivalently, to an excited state. In both cases the main ingredients for maintaining fibrillation, namely the presence of both propagating wavefronts and a sufficient mass of excitable tissue at rest, referred to as "excitable gap", in which these wavefronts can travel, are missing. Achieving these objectives is challenging since, on biophysical grounds, shock-induced changes in polarization of both polarities are always present during shock delivery.

The main ingredient in the optimal control approach is to design a proper cost functional. A natural choice in optimizing the process of defibrillation is to determine a control variable which aim to minimize undesired values of the transmembrane voltage, $v$, based on a given desired trajectory. In our computations, the cost functional is chosen as

$$
J\left(v, I_{e}\right)=J_{1}(v)+J_{2}\left(I_{e}\right),
$$

where $J_{1}(v)$ and $J_{2}\left(I_{e}\right)$ denote state cost and control cost, respectively. The extracellular current $I_{e}$ injected in and withdrawn from the extracellular domain is considered as control input. The transmembrane voltage $v$ is the state variable at the tissue domain which needs to be optimized based on a desired trajectory solution.

Solving optimal control problems with nonlinear PDEs as constraints poses a significant numerical challenge due to the large size of the state systems and the high spatio-temporal resolutions required to resolve the dynamics of wavefront propagation in the heart. Moreover, solving the optimality system involves solving both the primal as well as the adjoint equations. These two systems are of similar complexity and must be solved frequently within any iterative solution process. Therefore, efficient discretization techniques play an important role. Here we have chosen the finite element method for spatial discretization and higher order Rosenbrock time stepping methods for the temporal discretization to solve the PDE system.

Due to these high computational costs the application of parallel computing techniques is essential to enable large scale simulations of such PDE constrained optimal control problems on realistic geometries. In this study we applied a NewtonKrylov method to the Lagrangian stationary conditions. The implementation of the parallel optimization code was based on the freely available public domain package DUNE [4].

The outline of the paper is as follows. In the next section we briefly describe the bidomain model equations for cardiac tissue immersed in a conductive bath volume. A biventricular slice model is used for numerical experiments where tissue structure is assumed to be transversely isotropic and the regularized Mitchell-Schaeffer ionic model is used to describe cellular dynamics. In Section 3, the optimal control frame work is presented and the derivation of first and second order conditions is discussed. The numerical discretization of the optimality system and optimization algorithm to solve the discretized system is explained in Section 4. Numerical results are shown for the termination of reentrant waves in Section 5 . 


\section{Mathematical model}

The cardiac tissue and the surrounding bath domains are denoted by $\Omega_{H} \subset \mathbb{R}^{3}$, and $\Omega_{B} \subset \mathbb{R}^{3}$, respectively, and $\Omega=\bar{\Omega}_{H} \cup \Omega_{B}$. The space-time cylinder of the whole domain is denoted by $Q=\Omega \times(0, T]$ and the cardiac tissue and bath volume are expressed as $Q_{B}=\Omega_{B} \times(0, T]$ and $Q_{H}=\Omega_{H} \times(0, T]$.

The bidomain equations consist of the following coupled system of PDEs and ODEs together with initial and boundary conditions which will be determined below:

$$
\begin{aligned}
0 & =\nabla \cdot \bar{\sigma}_{b} \nabla u_{b} \quad \text { in } Q_{B} \\
0 & =\nabla \cdot\left(\bar{\sigma}_{i}+\bar{\sigma}_{e}\right) \nabla u_{e}+\nabla \cdot \bar{\sigma}_{i} \nabla v \quad \text { in } Q_{H} \\
\frac{\partial v}{\partial t} & =\nabla \cdot \bar{\sigma}_{i} \nabla v+\nabla \cdot \bar{\sigma}_{i} \nabla u_{e}-I_{i o n}(v, w)+I_{t r}(x, t) \quad \text { in } Q_{H} \\
\frac{\partial w}{\partial t} & =G(v, w) \quad \text { in } Q_{H},
\end{aligned}
$$

where $u_{b}: Q_{B} \rightarrow \mathbb{R}$ is the bath potential in the bath volume, $u_{e}: Q_{H} \rightarrow \mathbb{R}$ is the extracellular potential, $w: Q_{H} \rightarrow \mathbb{R}^{n}$ are the cell model state variables, $\overline{\sigma_{b}}: \Omega_{B} \rightarrow \mathbb{R}^{d \times d}$ the extracellular conductivity tensors in the bath volume, $\bar{\sigma}_{i}: \Omega_{H} \rightarrow \mathbb{R}^{d \times d}$ and $\bar{\sigma}_{e}: \Omega_{H} \rightarrow \mathbb{R}^{d \times d}$ are respectively the intracellular and extracellular conductivity tensors and the spatial dimension is $d=3$ here. The term $I_{t r}$ is the transmembrane current density stimulus as delivered by the intracellular electrode. Here, $v: Q_{H} \rightarrow \mathbb{R}$ denotes the transmembrane voltage which is the difference between the intracellular and extracellular potentials. Finally $I_{i o n}(v, w)$ is the current density flowing through the ionic channels and $G(v, w)$ determines the evolution of the gating variables, which are determined by an electrophysiological cell model, see e.g. [1] for description on these models. Eqs. (2)-(5) are referred to as primal system in the context of optimal control.

\section{Conductivity tensors}

The anisotropy within the intra and extracellular domains of the cardiac tissue is characterized by the conductivity tensors $\bar{\sigma}_{i}$ and $\bar{\sigma}_{e}$. It is a consequence of myocyte geometry, the spatial arrangement of myocytes and interstitial spaces, as well as the direction dependent expression of gap junction proteins which interconnect the intracellular spaces of adjacent myocytes. This yields conductivities which are higher along the long axes of myocytes, often referred to as "fiber orientation", than in the transverse direction. In principle the ventricular myocardium is also organized in sheets, which at any spatial point gives rise to three characteristic directions for the conductivity values of the tissue [33], parallel to the fibers, perpendicular to the fibers but parallel to the sheet, and perpendicular to the sheet. Let $\mathbf{a}_{l}(\mathbf{x}), \mathbf{a}_{t}(\mathbf{x})$ and $\mathbf{a}_{n}(\mathbf{x})$ be the orthonormal vectors associated to the structure of the myocardium at any spatial point $\mathbf{x}$ where $\mathbf{a}_{l}(\mathbf{x})$ is parallel to the fibers, $\mathbf{a}_{t}(\mathbf{x})$ is perpendicular to the fibers but parallel to the sheet plane, and $\mathbf{a}_{n}(\mathbf{x})$ is normal to the sheet plane. The measured conductivity coefficients along the corresponding directions are denoted by $\sigma_{l}^{i, e}, \sigma_{t}^{i, e}$ and $\sigma_{n}^{i, e}$. In this study the simplifying assumption of rotational isotropy is made, that is, $\sigma_{n}^{i, e}=\sigma_{t}^{i, e}$. Consequently, the local intracellular conductivity tensor $\bar{\sigma}^{i}$ is expressed as

$$
\bar{\sigma}^{i}=\left(\sigma_{l}^{i}-\sigma_{t}^{i}\right) \mathbf{a}_{l}(\mathbf{x}) \mathbf{a}_{l}^{T}(\mathbf{x})+\sigma_{t}^{i} I,
$$

where $I$ is the identity matrix. 


\section{Membrane model}

Cellular dynamics that we follow here is described by the model proposed by MitchellSchaeffer (MS) [27] and modified by Keener [24]

$$
\begin{aligned}
I_{i o n}(v, w) & =-\frac{1}{\tau_{\text {in }}} w m^{2}(v)\left[v_{p}-v\right]+\frac{1}{\tau_{\text {out }}}\left(v-v_{r}\right) \\
G(v, w) & =\alpha_{w}(v)[1-w]-\beta_{w}(v) w,
\end{aligned}
$$

where

$$
\begin{gathered}
m(v)= \begin{cases}0, & v<v_{r} \\
\frac{v-v_{r}}{v_{p}-v_{r}}, & v_{r}<v<v_{p} \\
1, & \text { else. }\end{cases} \\
\alpha_{w}(v)=\frac{1-f(v)}{\tau_{\text {open }}+\left(\tau_{\text {close }}-\tau_{\text {open }}\right) f(v)} \\
\beta_{w}(v)=\frac{f(v)}{\tau_{\text {open }}+\left(\tau_{\text {close }}-\tau_{\text {open }}\right) f(v)} \\
f(v)=\frac{1}{2}\left(1+\tanh \left(\kappa\left(v-v_{\text {gate }}\right)\right)\right) .
\end{gathered}
$$

Here $v_{r}$ is the resting potential, $v_{p}$ is the peak potential and $v_{\text {gate }}$ is the threshold voltage. The time constants $\tau_{\text {in }}$ and $\tau_{\text {out }}$ govern the length of depolarization and repolarization phases where $\tau_{\text {open }}$ and $\tau_{\text {close }}$ are the characteristic time constants of gate opening and closing, respectively. The above regularized MS model reduces to the original MS model if $\kappa \rightarrow \infty$. While the MS model consists of two state variables only which keeps the computational complexity as low as possible, it faithfully captures several important salient action potential features such as realistic restitution properties. The parameter values, see [27], for this model are given in Table 1.

\begin{tabular}{|cr|cr|}
\hline$\tau_{\text {in }}$ & 0.3 & $\kappa$ & 1000 \\
$\tau_{\text {out }}$ & 6.0 & $v_{\text {gate }}$ & -67 \\
$\tau_{\text {open }}$ & 120 & $v_{r}$ & -80 \\
$\tau_{\text {close }}$ & 150 & $v_{p}$ & 40 \\
\hline
\end{tabular}

Table 1: Simulation parameters.

\section{Boundary and initial conditions}

Here we assume that there is no intracellular current flow across the tissue-bath interface and thus homogeneous Neumann boundary condition are applied to seal the boundaries along the intracellular space,

$$
\eta \cdot\left(\bar{\sigma}_{i} \nabla v+\bar{\sigma}_{i} \nabla u_{e}\right)=0 \quad \text { on } \quad \Sigma_{H}=\partial \Omega_{H} \times(0, T],
$$

where $\eta$ is the outer unit normal vector to $\Omega_{H}$. The tissue-bath interface conditions in the extracellular domain were set up to enforce current conservation across the interface [39],

$$
\eta \cdot \bar{\sigma}_{e} \nabla u_{e}=\eta \cdot \overline{\sigma_{b}} \nabla u_{b} \text { on } \Sigma_{H}
$$


Moreover, the extracellular and the bath potentials must be continuous across the interface and therefore,

$$
u_{e}=u_{b} \text { on } \Sigma_{H}
$$

We assume zero flux at the boundary of the bath domain and apply homogeneous Neumann boundary conditions, except for those parts of the boundary where bath stimuli $\hat{I}_{e}(t)$ are applied:

$$
\begin{aligned}
& \eta \cdot \bar{\sigma}_{b} \nabla u_{b}=\hat{I}_{e}(t, x) \quad \text { on } \quad \Gamma_{12} \times(0, T] \\
& \eta \cdot \bar{\sigma}_{b} \nabla u_{b}=0 \quad \text { on } \partial \Omega_{B} \backslash \Gamma_{12} \times(0, T] .
\end{aligned}
$$

The current $\hat{I}_{e}$ acts as control along the boundary $\Gamma_{12}=\Gamma_{1} \cup \Gamma_{2}$, where $\Gamma_{i}, i=1,2,3$ are mutually disjoint and satisfy $\Gamma_{1} \cup \Gamma_{2} \cup \Gamma_{3}=\partial \Omega_{B}$, see Figure 1. For compatibility reasons it is assumed throughout that

$$
\int_{\Gamma 12} \hat{I}_{e}(t, \cdot) d s=0 \quad \forall t \in(0, T) .
$$

In the numerical experiments, $\hat{I}_{e}$ is only temporally dependent and will take the following form

$$
\hat{I}_{e}=I_{e}(t)\left(\chi_{\Gamma_{1}}-\chi_{\Gamma_{2}}\right),
$$

where $\chi_{\Gamma_{i}}$ is the characteristic function of the predefined set $\Gamma_{i}, i=1,2$. Then condition (18) is satisfied if $\left|\Gamma_{1}\right|=\left|\Gamma_{2}\right|$ where the support regions $\Gamma_{1}$ and $\Gamma_{2}$ can be considered to represent a cathode and an anode respectively. Moreover, we impose zero mean condition for the extracellular potential in order to obtain uniqueness of the elliptic systems:

$$
\int_{\Omega_{H}} u_{e}=0
$$

The initial values of the transmembrane voltage and cell model state variable are given by prescribed values.

$$
v(x, 0)=v_{0} \quad \text { and } \quad w(x, 0)=w_{0} \quad \text { on } \Omega,
$$

where $v_{0}: \Omega \rightarrow \mathbb{R}$ denotes the initial transmembrane potential and $w_{0}: \Omega \rightarrow \mathbb{R}$ is the initial gating variable at time $t=0$.

To conclude this section we provide some background on the well-posedness of the mathematical model. For this purpose we introduce

$$
V=\left\{\varphi \in H^{1}(\Omega): \int_{\Omega_{H}} \varphi d x=0\right\}, V_{H}=H^{1}\left(\Omega_{H}\right), H=L^{2}\left(\Omega_{H}\right)
$$

and set

$$
\bar{\sigma}=\left\{\begin{array}{r}
\overline{\sigma_{b}} \text { in } \Omega_{B} \\
\overline{\sigma_{i}}+\overline{\sigma_{e}} \text { in } \Omega_{H} .
\end{array} \quad u=\left\{\begin{array}{l}
u_{b} \text { in } \Omega_{B} \\
u_{e} \text { in } \Omega_{H} .
\end{array}\right.\right.
$$

Definition 2.1. A triple $(u, v, w) \in L^{\infty}(0, T ; V) \times\left(L^{\infty}\left(0, T ; V_{H}\right) \cap H^{1}(0, T ; H)\right) \times$ $H^{1}(0, T ; H)$ is called variational solution to the bidomain equations $(2)-(5),(13)-(18)$ if (20) holds, and

$$
\int_{\Omega_{H}} \overline{\sigma_{i}} \nabla v(t) \nabla \varphi d x+\int_{\Omega} \bar{\sigma} \nabla u(t) \nabla \varphi d x=\int_{\partial \Omega_{B}} \hat{I}_{e}(t) \varphi d s,
$$




$$
\begin{gathered}
\int_{\Omega_{H}} v_{t}(t) \psi d x+\int_{\Omega_{H}} \overline{\sigma_{i}} \nabla\left(u_{e}(t)+v(t)\right) \nabla \psi d x+\int_{\Omega_{H}} I_{\text {ion }}(v(t), w(t)) \psi d x=\int_{\Omega_{H}} I_{t r}(t) \psi d x \\
w_{t}(t)-G(v(t), w(t))=0,
\end{gathered}
$$

for all $(\varphi, \psi) \in V \times V_{H}$. Here equations (21) and (22) are supposed to hold almost every where with respect to $t \in(0, T)$ and $(23)$ a.e. in $\Omega_{H} \times(0, T]$.

The following existence result is verified in [12] for the homogenous case, but the inclusion of the forcing term $\hat{I}_{e}$ can be achieved by standard methods.

Proposition 2.2. If $\hat{I}_{e} \in L^{2}\left(\partial \Omega_{B} \times(0, T)\right), I_{t r} \in L^{2}\left(\Omega_{H} \times(0, T)\right)$,

$$
\overline{\sigma_{i}} \in L^{\infty}\left(\Omega_{H}\right)^{d \times d}, \overline{\sigma_{e}} \in L^{\infty}\left(\Omega_{H}\right)^{d \times d}, \overline{\sigma_{b}} \in L^{\infty}\left(\Omega_{B}\right)^{d \times d},
$$

are symmetric and uniformly elliptic, and if $v_{0} \in V_{H}$, and $w_{0} \in L^{\infty}\left(\Omega_{H}\right)$, with $r<w_{0} \leq 1$ in $\Omega_{H}$, for some $r>0$, then (2)-(5), (13)-(18) admits a variational solution.

\section{The optimal control problem}

In this section we discuss the optimal control formulation for cardiac defibrillation that we follow here. The goal consists in finding an extracellular current $I_{e}(t)$ which is as weak as possible while still leading to defibrillation. This will be achieved by means of minimizing a cost-functional subject to the bidomain equations. More precisely, the cardiac defibrillation will aim at driving the transmembrane voltage $v$ to a desired value denoted by $v_{d}$ at the intracellular space by properly applying the $I_{e}$ at the boundary of bath domain. The resulting optimization problem becomes,

$$
\left\{\begin{array}{l}
\min J\left(v, I_{e}\right)=\min \frac{1}{2} \int_{0}^{T}\left(\alpha_{1} \int_{\Omega_{H}}\left|v-v_{d}\right|^{2} \mathrm{~d} x+\alpha_{2}\left|I_{e}(t)\right|^{2}\right) \mathrm{d} t, \\
\text { subject to }(2)-(5) \text { and } I_{e} \in U=L^{2}(0, T ; \mathbb{R}),
\end{array}\right.
$$

Here $\alpha_{1}$ and $\alpha_{2}$ denote positive weights for the tracking term and the control cost respectively. It can be shown with standard techniques that there exists an optimal input $I_{e}^{\star}$ with an associated state variables $\left(u^{\star}, v^{\star}, w^{\star}\right)$ which solve $(24)$. The numerical realization of (24) relies on the necessary optimality conditions which are obtained by applying a formal Lagrange formulation. For this purpose we define the Lagrangian corresponding to (24) by

$$
\begin{aligned}
& \mathcal{L}\left(u_{b}, u_{e}, v, w, I_{e}, p_{b}, p, q, r\right)=J\left(v, I_{e}\right) \\
& -\int_{0}^{T} \int_{\Omega} \sigma_{b} \nabla u_{b} \nabla p_{b}+\int_{0}^{T} \int_{\Gamma_{12}} \hat{I}_{e} p_{b} \mathrm{~d} s \mathrm{~d} t \\
& -\int_{0}^{T} \int_{\Omega}\left(\sigma_{i} \nabla v \nabla p_{e}+\left(\sigma_{i}+\sigma_{e}\right) \nabla u_{e} \nabla p_{e}\right) \mathrm{d} x \mathrm{~d} t \\
& -\int_{0}^{T} \int_{\Omega}\left(v_{t} q+\sigma_{i}(\nabla u+\nabla v) \nabla q+I_{\text {ion }}(v, w) q-I_{t r} q\right) \mathrm{d} x \mathrm{~d} t \\
& -\int_{0}^{T} \int_{\Omega} w_{t} r-G(v, w) r \mathrm{~d} x \mathrm{~d} t,
\end{aligned}
$$


where $p_{b}, p_{e}, q, r$ are the Lagrange multipliers associated to the state Eqs. (2)-(5). The first order optimality system is given by the Karusch-Kuhn-Tucker (KKT) conditions. For this purpose the partial derivatives of $\mathcal{L}$ with respect to $u_{b}, u_{e}, v$ and $w$ are set equal to zero. We obtain the following system of equations which are refer to as the dual equations,

$$
\begin{aligned}
0 & =\nabla \cdot\left(\bar{\sigma}_{i}+\bar{\sigma}_{e}\right) \nabla p_{e}+\nabla \cdot \bar{\sigma}_{i} \nabla q \text { in } Q_{H}, \\
\frac{\partial q}{\partial t} & =-\nabla \cdot \bar{\sigma}_{i} \nabla p_{e}-\nabla \cdot \bar{\sigma}_{i} \nabla q+\left(I_{i o n}\right)_{v} q-(G)_{v} r-\left.\left(V_{m}-V_{d}\right)\right|_{\Omega_{o b s}} \text { in } Q_{H}, \\
\frac{\partial r}{\partial t} & =\left(I_{i o n}\right)_{w} q-(G)_{w} r \text { in } Q_{H}, \\
0 & =\nabla \cdot \bar{\sigma}_{b} \nabla p_{b} \quad \text { in } Q_{B},
\end{aligned}
$$

where $p_{b}, p_{e}, q$ and $r$ are the Lagrange multipliers associated to $u_{b}, u_{e}, v$ and $w$ respectively. The terminal conditions are

$$
q(T)=0, \quad r(T)=0,
$$

and the boundary conditions for the adjoint states must satisfy

$$
\begin{aligned}
\eta \cdot\left(\bar{\sigma}_{i} \nabla p_{e}+\bar{\sigma}_{i} \nabla q\right)=0 & \text { on } \Sigma_{H} \\
\overline{\sigma_{b}} \nabla p_{b} \cdot \eta=0 & \text { on } \partial \Omega_{B} \times[0, T] .
\end{aligned}
$$

Furthermore, the following interface conditions must be observed:

$$
p_{b}=p_{e} \text { and } \sigma_{i} \nabla p_{e}=\sigma_{b} \nabla p_{b} \text { on } \Sigma_{H} .
$$

In addition the zero mean conditions $\int_{\Omega_{H}} p_{e}(t) d x=0$ holds for all $t \in(0, T)$. Finally we have the optimality condition:

$$
\left(\alpha I_{e}^{*}(t)+\int_{\Gamma_{1}} p_{b}(x, t) d x-\int_{\Gamma_{2}} p_{b}(x, t) d x\right)\left(I_{e}(t)-I_{e}^{*}(t)\right) \geq 0 \quad \text { for all } I_{e} \in U
$$

for almost every $t \in(0, T)$ and the minimizer $I_{e}^{*}$. In case, the constraints are not active this inequality results in

$$
\alpha I_{e}^{*}(t)+\int_{\Gamma_{1}} p_{b}(x, t) d x-\int_{\Gamma_{2}} p_{b}(x, t) d x=0 \text {, for a.e. } t \in(0, T) .
$$

\section{Newton's method}

Let us explain Newton's method to solve the optimization problem. It is based on the reduced cost functional $\hat{J}\left(I_{e}\right)=J\left(v\left(I_{e}\right), I_{e}\right)$, where $v\left(I_{e}\right)$ denotes the second coordinate of the variational solution $\left(u\left(I_{e}\right), v\left(I_{e}\right), w\left(I_{e}\right)\right)$ as a function of $I_{e}$. Thus $\hat{J}\left(I_{e}\right)$ involves implicitly the state variables depending on $I_{e}$. The Newton's method aims for computing $I_{e}^{\star}$ by updating $I_{e}^{k}$ in an iterative fashion based on the following system:

$$
\hat{J}^{\prime \prime}\left(I_{e}^{k}\right) \delta I_{e}=-\nabla \hat{J}\left(I_{e}^{k}\right), \quad I_{e}^{k+1}:=I_{e}^{k}+\beta \delta I_{e},
$$

where $\hat{J}^{\prime \prime}\left(I_{e}^{k}\right)$ denotes the Hessian of the reduced cost functional, $\delta I_{e}$ is the search direction obtained from the Newton equation and $\beta$ is the step length parameter attained from the line search method. For large-scale PDE-constrained optimization 
problems like the present one, explicit construction of the Hessian matrix is not feasible. Moreover, solving Eq. (30) with a direct method is generally impractical. Therefore, an inexact-Newton-Krylov approach is used to solve the system (30). Due to the attractive use of matrix-vector products Krylov based solvers avoid the need to form the Hessian matrix explicitly. We briefly summarize the main ingredients to compute "the action of the Hessian of the reduced cost" on a given vector.

For brevity, the state variables on bath and tissue domains are denoted by $y=$ $\left(u_{b}, u_{e}, v, w\right)$. The second derivative of the reduced cost functional is derived based on the Lagrangian functional which can be expressed as follows, see for more details $[13,16]$.

$$
\hat{J}^{\prime \prime}\left(I_{e}\right)=\mathcal{L}_{I_{e} I_{e}}+\delta y^{*} \mathcal{L}_{y I_{e}}+\mathcal{L}_{I_{e} y} \delta y+\delta y^{*} \mathcal{L}_{y y} \delta y,
$$

where $\delta y=-e_{y}^{-1} e_{I_{e}}$. Here $e=e\left(u_{b}, u_{e}, v, w, I_{e}\right)$ is an abstract notation for the complete coupled PDE and ODE bidomain system (2-5) together with boundary and initial conditions. Given $\mathbf{I}_{e}$, then considering $e\left(u_{b}, u_{e}, v, w, I_{e}\right)=0$ means solving the primal (nonlinear) equations for $\left(u_{b}, u_{e}, v, w\right)$ as a function of $I_{e}$. Next, $e_{y}$ denotes the linearized primal system where we have set $y=\left(u_{b}, u_{e}, v, w\right)$, and $e_{y}^{-1} \tilde{f}$ stands for solving the linearized primal system for some right hand side $\tilde{f}$. The derivatives are always taken at a current iteration point $\left(u_{b}\left(\left(I_{e}\right)_{k}\right), u_{e}\left(\left(I_{e}\right)_{k}\right), v\left(\left(I_{e}\right)_{k}\right), w\left(\left(I_{e}\right)_{k}\right),\left(I_{e}\right)_{k}\right)$ of the Newton method. Further, to compute $\hat{J}$ the second derivative of the operator $\mathcal{L}$ applied to the vector $\left(\delta u_{b}, \delta u_{e}, \delta v, \delta w\right)$ is needed. It can be expressed as follows

$$
\mathcal{L}_{y y}=\left(\begin{array}{c}
0 \\
0 \\
\left.\delta v\right|_{\Omega o b s}-\left[I_{i o n}\right]_{v v} q \delta v-\left[I_{i o n}\right]_{v w} q \delta w+[G]_{v v} r \delta v+[G]_{v w} r \delta w \\
-\left[I_{i o n}\right]_{w v} q \delta v-\left[I_{i o n}\right]_{w w} q \delta w+[G]_{w v} r \delta v+[G]_{w w} r \delta w
\end{array}\right)
$$

where the subscripts denote the derivatives w.r.t. the state variables. To compute the action of the Hessian on a given vector requires to compute the dual-(or adjoint) equation to obtain the action of $\delta y^{*}=-e_{I_{e}}^{*}\left(e_{y}^{-1}\right)^{*}$. In fact, every step of a Krylov solver to solve (30) requires one linearized primal (contained in $\delta y$ ) and dual solve (contained in $\delta y^{*}$ ) For detailed exposition of the Newton's algorithm for the bidomain equations we refer to [16, Section 2]

\section{Numerical approach}

Here we briefly explain the space and time discretization techniques to solve the partial differential equations which are needed to resolve the complete optimality system. A finite element method was used for the spatial discretization and a linearly implicit Runge-Kutta methods for the temporal discretizations.

\section{Semi-discretization in space}

In this subsection we give an overview of the spatial discretization of the primal and dual equations by a piecewise bilinear finite element method based on the weak formulation.

\section{Space discretization of the primal problem}

In our computations, the elliptic system on the bath domain Eq. (2) and on the tissue domain Eq. (3) can be solved monolithically. Here we define $u$ as the extracellular 
potential on $\Omega_{H} \cup \Omega_{B}$, i.e.:

$$
u= \begin{cases}u_{b} & \text { in } \Omega_{B} \\ u_{e} & \text { in } \Omega_{H}\end{cases}
$$

and introduce the global conductivity tensor

$$
\bar{\sigma}= \begin{cases}\bar{\sigma}_{b} & \text { in } \Omega_{B} \\ \left(\bar{\sigma}_{i}+\bar{\sigma}_{e}\right) & \text { in } \Omega_{H}\end{cases}
$$

The weak solution $(u, v, w) \in V \times V_{H} \times H$ of the combined system satisfies for all $(\varphi, \psi) \in V \times V_{H}$

$$
\begin{aligned}
\int_{\Omega_{H}} \bar{\sigma}_{i} \nabla v(t) \nabla \varphi \mathrm{d} x+\int_{\Omega} \bar{\sigma} \nabla u(t) \nabla \varphi \mathrm{d} x & =\int_{\partial \Omega_{B}} \hat{I}_{e}(t, \cdot) \varphi_{b} \mathrm{~d} x \\
\left\langle v_{t}(t), \psi\right\rangle_{V^{*}, V}+\int_{\Omega_{H}} \bar{\sigma}_{i} \nabla\left(u_{e}(t)+v(t)\right) \nabla \psi d x & +\int_{\Omega_{H}} I_{i o n}(v(t), w(t)) \psi d x \\
& =\left\langle I_{t r}(t), \psi\right\rangle_{V^{*}, V}, \\
w_{t}(t)=G(v(t, x), w(t, x)) \text { a.e. in } \Omega_{H}, &
\end{aligned}
$$

together with initial and boundary conditions (13)-(20). Let $V^{h} \subset H^{1}\left(\Omega_{H}\right)$ be the finite dimensional subspace of continuous piecewise linear basis functions with respect to the spatial grid. The approximate solutions $\mathbf{u}, \mathbf{v}$ and $\mathbf{w}$ are expressed in the form $\mathbf{u}(t)=\sum_{i=0}^{M+N} u_{i}(t) \omega_{i}, \mathbf{v}(t)=\sum_{i=0}^{N} v_{i}(t) \omega_{i}$ and $\mathbf{w}(t)=\sum_{i=0}^{N} w_{i}(t) \omega_{i}$, respectively, where $\left\{\omega_{i}\right\}_{i=1}^{N}$ and $\left\{\omega_{i}\right\}_{i=N+1}^{M}$ denote the basis functions. This semidiscretization in space results in the differential algebraic system:

$$
\begin{aligned}
\mathbf{A}_{\mathbf{i e}} \mathbf{u}+\mathbf{A}_{\mathbf{i}} \mathbf{v} & =\mathbf{I}_{e} \\
\mathbf{M} \frac{\partial \mathbf{v}}{\partial t} & =-\mathbf{A}_{\mathbf{i}} \mathbf{v}-\mathbf{A}_{\mathbf{i}} \mathbf{u}-\mathbf{I}_{i o n}(\mathbf{v}, \mathbf{w})+\mathbf{I}_{t r} \\
\mathbf{M} \frac{\partial \mathbf{w}}{\partial t} & =\mathbf{G}(\mathbf{v}, \mathbf{w}),
\end{aligned}
$$

together with initial conditions for $\mathbf{v}$ and $\mathbf{w}$, where $\mathbf{A}_{\mathbf{i e}}=\left\{\left\langle\left(\sigma_{i}+\sigma_{e}\right) \nabla \omega_{i}, \nabla \omega_{j}\right\rangle_{\Omega}\right\}_{i, j=1}^{M+N}$ and $\mathbf{A}_{\mathbf{i}}=\left\{\left\langle\sigma_{i} \nabla \omega_{i}, \nabla \omega_{j}\right\rangle_{\Omega_{H}}\right\}_{i, j=1}^{N}$ are the stiffness matrices, $\mathbf{M}=\left\{\left\langle\omega_{i}, \omega_{j}\right\rangle_{\Omega_{H}}\right\}_{i, j=1}^{N}$ is the mass matrix, the vectors $\mathbf{I}_{e}, \mathbf{I}_{i t r}$ are defined by $\mathbf{I}_{e}=\left\{\left\langle\left(\chi_{\Gamma_{1}} I_{e}-\chi_{\Gamma_{2}} I_{e}\right), \omega_{j}\right\rangle_{\Omega}\right\}_{j=1}^{M_{\Gamma}}$ and $\mathbf{I}_{t r}=\left\{\left\langle I_{t r}, \omega_{j}\right\rangle_{\Omega_{H}}\right\}_{j=1}^{N}$, respectively. The expressions $\left(\mathbf{I}_{i o n}\right)(\mathbf{v}, \mathbf{w})$ and $\mathbf{G}(\mathbf{v}, \mathbf{w})$ are defined by

$$
\begin{aligned}
\mathbf{I}_{\text {ion }}(\mathbf{v}, \mathbf{w}) & =\left\{I_{\text {ion }}\left(\sum_{i=0}^{N} v_{i} \omega_{i}, \sum_{i=0}^{N} w_{i} \omega_{i}\right), \omega_{j}\right\}_{j=1}^{N}, \\
\mathbf{G}(\mathbf{v}, \mathbf{w}) & =\left\{G\left(\sum_{i=0}^{N} v_{i} \omega_{i}, \sum_{i=0}^{N} w_{i} \omega_{i}\right), \omega_{j}\right\}_{j=1}^{N} .
\end{aligned}
$$

\section{Space discretization of the dual problem}

We use the same finite element space to discretize the dual equations in the bath and tissue domains. The approximate solutions $\mathbf{p}, \mathbf{q}$, and $\mathbf{r}$ can be expressed in the form 
$\mathbf{p}(t)=\sum_{i=0}^{M+N} p_{i}(t) \omega_{i}, \mathbf{q}(t)=\sum_{i=0}^{N} q_{i}(t) \omega_{i}$ and $\mathbf{r}(t)=\sum_{i=0}^{N} r_{i}(t) \omega_{i}$ respectively and the following semi discrete form of the dual equations is obtained:

$$
\begin{aligned}
\mathbf{A}_{\mathbf{i e}} \mathbf{p}+\mathbf{A}_{\mathbf{i}} \mathbf{q} & =0 \\
\mathbf{M} \frac{\partial \mathbf{q}}{\partial t} & =\mathbf{A}_{\mathbf{i}} \mathbf{p}+\mathbf{A}_{\mathbf{i}} \mathbf{q}+\left(\mathbf{I}_{i o n}\right)_{\mathbf{v}}(\mathbf{v}, \mathbf{w})-\mathbf{G}_{v}(\mathbf{v}, \mathbf{w}) \mathbf{M r}-\mathbf{M}_{o b s}\left(\mathbf{v}-\mathbf{v}_{d}\right) \\
\mathbf{M} \frac{\partial \mathbf{r}}{\partial t} & =-\mathbf{G}_{w}(\mathbf{v}, \mathbf{w}) \mathbf{r}+\left(\mathbf{I}_{i o n}\right)_{\mathbf{w}}(\mathbf{v}, \mathbf{w}) \mathbf{q}
\end{aligned}
$$

with terminal conditions $\mathbf{q}(T)=0, \mathbf{r}(T)=0$. The locally defined mass matrix for the observation domain is $\mathbf{M}_{o b s}=\left\{\left\langle\omega_{i}, \chi_{\Omega_{o b s}} \omega_{j}\right\rangle\right\}_{i, j=1}^{N}$. The expressions $\left(\mathbf{I}_{i o n}\right)_{\mathbf{v}}(\mathbf{v}, \mathbf{w})$, $\left(\mathbf{I}_{i o n}\right)_{\mathbf{w}}(\mathbf{v}, \mathbf{w})\left(\mathbf{G}_{i o n}\right)_{\mathbf{v}}(\mathbf{v}, \mathbf{w})$ and $\left(\mathbf{G}_{i o n}\right)_{\mathbf{w}}(\mathbf{v}, \mathbf{w})$ are defined by

$$
\begin{aligned}
\left(\mathbf{I}_{i o n}\right)_{\mathbf{v}}(\mathbf{v}, \mathbf{w}) & =\left\{\frac{\partial I_{i o n}}{\partial v}\left(\sum_{i=0}^{N} v_{i} \omega_{i}, \sum_{i=0}^{N} w_{i} \omega_{i}\right), \omega_{j}\right\}_{j=1}^{N} \\
\left(\mathbf{I}_{i o n}\right)_{\mathbf{w}}(\mathbf{v}, \mathbf{w}) & =\left\{\frac{\partial I_{i o n}}{\partial w}\left(\sum_{i=0}^{N} v_{i} \omega_{i}, \sum_{i=0}^{N} w_{i} \omega_{i}\right), \omega_{j}\right\}_{j=1}^{N} \\
(\mathbf{G})_{\mathbf{v}}(\mathbf{v}, \mathbf{w}) & =\left\{\frac{\partial G}{\partial v}\left(\sum_{i=0}^{N} v_{i} \omega_{i}, \sum_{i=0}^{N} w_{i} \omega_{i}\right), \omega_{j}\right\}_{j=1}^{N} \\
(\mathbf{G})_{\mathbf{w}}(\mathbf{v}, \mathbf{w}) & =\left\{\frac{\partial G}{\partial w}\left(\sum_{i=0}^{N} v_{i} \omega_{i}, \sum_{i=0}^{N} w_{i} \omega_{i}\right), \omega_{j}\right\}_{j=1}^{N} .
\end{aligned}
$$

Remark 4.1. We mention that $\mathbf{u}_{0}$ has zero mean and the compatibility condition for the singular algebraic system Eq. (37) is satisfied at each time iteration level, i.e.

$$
\mathbf{c}^{T}\left(-\mathbf{A}_{\mathbf{i}} \mathbf{v}-\mathbf{I}_{e}\right)=-\left(\mathbf{c}^{T} \mathbf{A}_{\mathbf{i}}\right) \mathbf{v}-\mathbf{c}^{T} \mathbf{I}_{e}=0,
$$

where $\mathbf{c}=(1, \ldots, 1)^{T}$ is a constant vector. Correspondingly, at every time-level the iterative procedure for solving (40) is initialized by a zero-mean function. Then the compatibility condition for the singular algebraic system (40) is satisfied.

Analogously, the same spatial discretization technique is applied for the linearized primal and dual equations.

\section{Time discretization}

In this subsection we give a brief description of the time discretization for solving the systems of ordinary differential equations. We solve the Eqs. (38) and (39) as a coupled system which can be expressed in the following form:

$$
\tilde{\mathbf{M}} \frac{\partial \mathbf{x}}{\partial t}=\mathbf{F}(\mathbf{x}), \quad \mathbf{x}\left(t^{0}\right)=\mathbf{x}^{\mathbf{0}},
$$

where

$$
\begin{aligned}
\tilde{\mathbf{M}} & =\left(\begin{array}{cc}
\mathbf{M} & 0 \\
0 & \mathbf{M}
\end{array}\right), \quad \mathbf{x}=\left(\begin{array}{c}
\mathbf{v} \\
\mathbf{w}
\end{array}\right) \\
\mathbf{F} & =\left(\begin{array}{c}
-\mathbf{A}_{\mathbf{i}} \mathbf{v}-\mathbf{A}_{\mathbf{i}} \mathbf{u}-\mathbf{I}_{i o n}(\mathbf{v}, \mathbf{w})+\mathbf{I}_{t r} \\
\mathbf{G}(\mathbf{v}, \mathbf{w}) .
\end{array}\right.
\end{aligned}
$$


Similarly, we can write the Eqs. (41) and (42) in the (43) form. To solve (43), we introduce discrete steps $0=t^{0}, t^{1}, \ldots, t^{n}=T$ in the time interval $[0, T]$ which are not necessarily equidistant. We further set $\tau^{i}=t^{i+1}-t^{i}$ and denote by $\mathbf{x}^{i}$ the numerical solution at time $t^{i}$. Rosenbrock type linearly implicit Runge-Kutta methods are used for time discretization. They belong to a large class of methods which try to avoid the nonlinear system and replace it by a sequence of linear ones. In our computations, we used exact derivatives of the vector $\mathbf{F}(\mathbf{x})$ for the construction of the Jacobian matrix. The ROS3PL method was employed which has four internal stages to solve in each time step see [25]. Here we do not repeat the time discretization details for the current problem which were well explained in [14, Section 3.2].

\section{Parallel implementation}

It is well known that due to complex geometries and space-time scaling considerations bidomain equations simulations are computationally challenging to achieve realistic simulations on the one hand and acceptable computational times on the other, see e.g. $[32,42,31,29]$. In our simulations the software package DUNE [4], especially the dune-pdelab [5] discretization module, which is a $\mathrm{C}++$ template based programming environment for solving a general class of PDE's was used. For parallel grid construction the ALUGrid library [18] was employed which in turn uses the METIS[23] graph partitioner for the decomposition of the grid. We briefly point out the need of partitioning the computational domain for the current problem.

The combined elliptic system Eq. 37 should be solved on the entire body domain $\Omega$ while the parabolic-ode system Eq. 43 needs to be solved on the cardiac tissue domain $\Omega_{H}$ only. We use the following partitioning strategy. First we partition the cardiac tissue $\Omega_{H}$ into $P$ nonoverlapping subdomains. Then the whole domain $\Omega$ is partitioned into $P$ nonoverlapping subdomains. Because of the domain decomposition of the disjoint domains (the whole domain and the tissue domain), each single processor assigned to two different subdomains one from the whole domain and one from tissue domain, which might be distinctive. Due to the presence of two grids, inter-communication between the processors is necessary at each time step. To accomplish the necessary communication a projection operator, realized as a generalized scattering operation, was used to map between the parallel data vectors $V_{m}$ and $\phi_{e}$.

\section{Solution procedure for the primal system}

We briefly turn to the solution procedure for the primal system. Analogous techniques were used to solve the dual as well as the linearized primal systems. After the time discretization of (43) we obtain a system of linear algebraic equations at each internal stage of the ROS3PL method. To solve this linear system we employed a BiCGSTAB method with Jacobi preconditioning.

The solutions of the singular linear systems which arise after the full discretization of Eq (3) is defined up to an additive constant. A zero mean condition is applied to fix this constant. For the numerical realization of this condition, we employed a stabilized saddle point formulation from the work of Bochev and Lehoucq [10] For the discussion and implementation details of this technique for the current problem we refer to $[14,16]$. Finally, to solve the linear system we employed a BiCGSTAB [40] method with AMG preconditioner [9], which is developed using a greedy heuristic 
algorithm for the aggregation based on a strength of connection criterion.

Summarizing, the algorithmic solution procedure to solve the primal system is the following one.

Step 1: Copy the transmembrane solution from the tissue domain ( $\mathrm{x}$ on $\Omega_{H}$ ) to the complete domain $\left(\mathbf{x}_{b}\right.$ on $\Omega$ ) by using inter-processor communication, as explained in the subsection parallel implementation at section 4 . Here the columns and rows of zeros are padded in the solution vector $\mathbf{x}_{b}$ at the bath domain.

Step 2: Use the solution $\mathrm{x}_{b}^{i}$ at time $t^{i}$, solve the discretized elliptic system (37) for $\mathbf{u}^{i+1}$ at time $t^{i+1}$ by using the stabilized saddle point approach.

Step 3: Communicate the extracellular potential solution from the complete domain $(\mathbf{u}$ on $\Omega)$ to the cardiac tissue domain $\left(\mathbf{u}_{e}\right.$ on $\left.\Omega_{H}\right)$ at time $t^{i+1}$.

Step 4: Finally, by utilizing the computed solutions $\mathbf{u}_{e}^{i+1}$ solve the discretized parabolic equation (38) for $\mathbf{x}^{i+1}$ at time $t^{i+1}$ by applying the linearly implicit Runge-Kutta method.

\section{Optimization algorithm}

Here we briefly explain the algorithm which is used to solve the complete optimality system. The essential component in the optimization algorithm is the action of the Hessian evaluation, see left hand side of Eq. (30), which needs to be evaluated at every inner iteration of the CG method. We note that in order to evaluate the action of the Hessian on a given vector, one linearized primal problem and one adjoint equation have to be solved, this is explained in step 2 below. The essential steps of the optimization algorithm are summarized as follows.

1. Compute the first derivative $\hat{J}^{\prime}\left(I_{e}^{k}\right)=\alpha I_{e}^{k}(t)+\int_{\Gamma_{1}} p_{b}(x, t) d x-\int_{\Gamma_{2}} p_{b}(x, t) d x$, which requires one primal and one adjoint solve.

2. In each CG iteration step for solving (30), evaluate the action of $\hat{J}^{\prime \prime}\left(I_{e}^{n}\right)$ on $\delta I$ by means of the following sequence of computation:

(a) solve the linearized primal equation for $\left(\delta u_{b}, \delta u_{e}, \delta v, \delta w\right)$ using $\delta I$

$$
\left(\begin{array}{c}
\nabla \cdot \sigma_{b} \nabla \delta u_{b} \\
\nabla \cdot\left(\sigma_{i}+\sigma_{e}\right) \nabla \delta u_{e}+\nabla \cdot\left(\sigma_{i} \nabla \delta v\right) \\
\nabla \cdot\left(\sigma_{i} \nabla \delta v\right)+\nabla \cdot\left(\sigma_{i} \nabla \delta u\right)-\left(\delta v_{t}+\left[I_{i o n}\right]_{v} \delta v+\left[I_{i o n}\right]_{w} \delta w\right) \\
\delta w_{t}-\frac{\eta_{2}}{v_{p}} \delta v+\eta_{2} \eta_{3} \delta w
\end{array}\right)=\left(\begin{array}{l}
0 \\
0 \\
0 \\
0
\end{array}\right)
$$

with the following initial, boundary and interface conditions

$$
\begin{aligned}
\eta \cdot\left(\sigma_{i} \nabla \delta v+\sigma_{i} \nabla \delta u\right) & =0 \text { on } \Sigma_{H} \\
\eta \cdot \sigma_{b} \nabla \delta u_{b} & =\left(\chi_{c_{\Gamma_{1}}}-\chi_{c_{\Gamma_{2}}}\right) \delta I(t) \quad \text { on } \Gamma_{12} \\
\eta \cdot \sigma_{b} \nabla \delta u_{b} & =0 \quad \text { on } \partial \Omega_{B} \backslash \Gamma_{12} \\
\eta \cdot \overline{\sigma_{e}} \nabla \delta u_{e} & =\eta \cdot \overline{\sigma_{b}} \nabla \delta u_{b} \text { and } \delta u_{e}=\delta u_{b} \text { on } \Sigma_{H} \\
\delta v(x, 0)=\delta v_{0} & \text { and } \delta w(x, 0)=\delta w_{0} \quad \text { on } \Omega,
\end{aligned}
$$

(b) evaluate $\left(\xi_{1}, \xi_{2}, \xi_{3}, \xi_{4}\right):=\mathcal{L}_{y y}\left(y^{k}, z^{k}\right)\left(\delta u_{b}, \delta u_{e}, \delta v, \delta w, \delta I_{e}\right)$ from (31), 


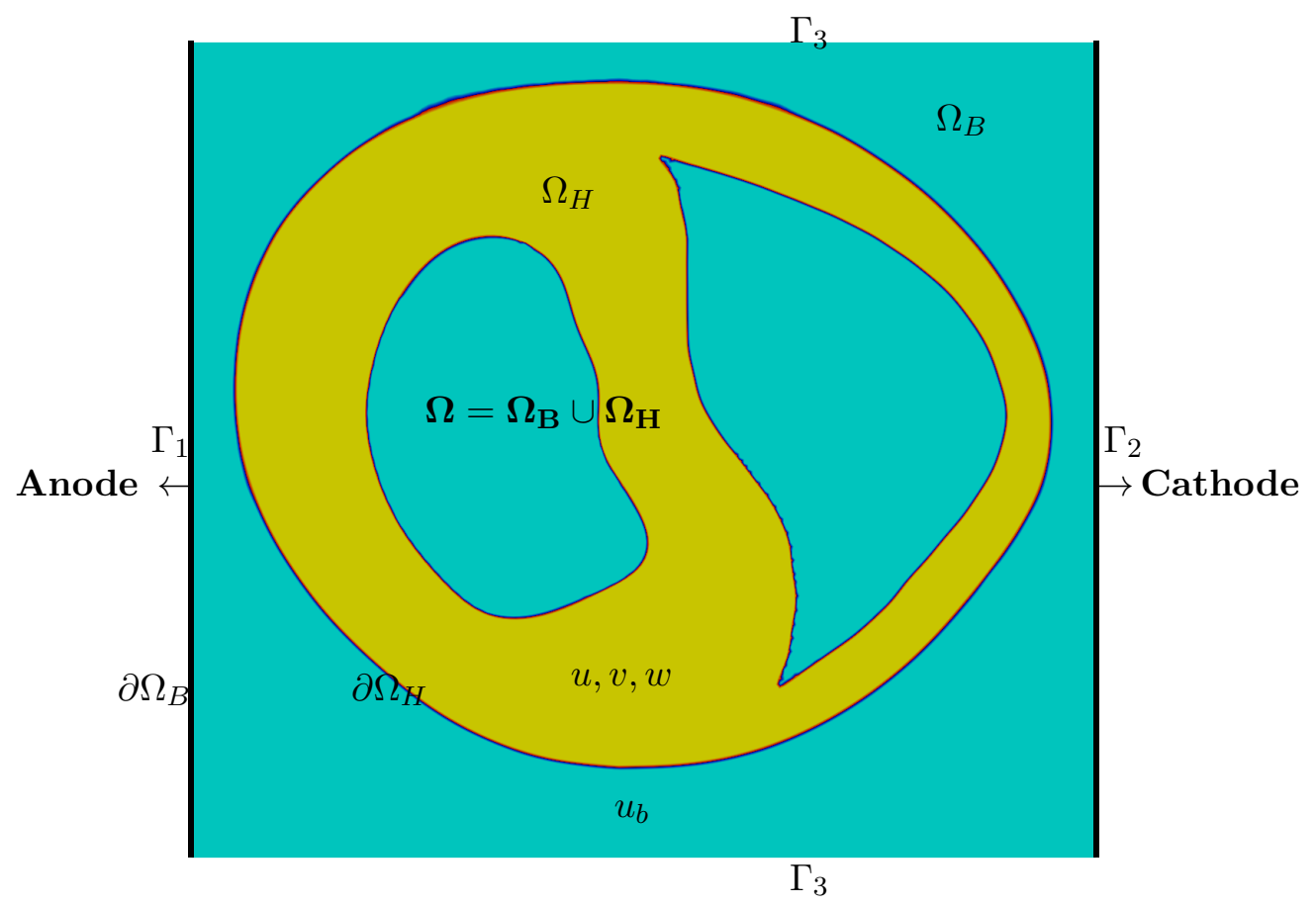

Figure 1: Schematic illustration of the computational domain which is comprised of tissue and bath domain.

(c) solve the adjoint equation with $\left(\xi_{1}, \xi_{2}, \xi_{3}, \xi_{4}\right)$ as r.h.s., i.e.

$$
\left(\begin{array}{c}
\nabla \cdot \sigma_{b} \nabla w_{1} \\
\nabla \cdot\left(\sigma_{i}+\sigma_{e}\right) \nabla w_{2}+\nabla \cdot \sigma_{i} \nabla w_{3} \\
\nabla \cdot \sigma_{i} \nabla w_{2}+\nabla \cdot \sigma_{i} \nabla w_{3}+w_{3 t}-\left[I_{i o n}\right]_{v} w_{3}-\frac{\eta_{2}}{v_{p}} w_{4} \\
-\left[I_{\text {ion }}\right]_{w} w_{3}-w_{4_{t}}+\eta_{2} \eta_{3} w_{4}
\end{array}\right)=\left(\begin{array}{c}
\xi_{1} \\
\xi_{2} \\
\xi_{3} \\
\xi_{4}
\end{array}\right)
$$

by using homogenous initial and boundary conditions,

(d) compute the action $\hat{J}^{\prime \prime}\left(I_{e}^{k}\right) \delta I=\left.\alpha \delta I\right|_{\Gamma_{12}}+\left.w_{1}\right|_{\Gamma_{1}}-\left.w_{1}\right|_{\Gamma_{2}}$.

\section{$5 \quad$ Numerical Results}

Numerical results for the optimization of cardiac defibrillation shocks based on the NCG and Newton's optimization algorithm are presented. For computations, a $3 \mathrm{D}$ slice geometry is considered which is generated from the histological image of rabbit ventricle [41]. The integrated domain $\Omega=\Omega_{H} \cup \Omega_{B}$ is $[-0.171,-0.121] \times$ $[-1.18,2.04] \times[-1.52,1.38]$ of size $0.05 \times 3.22 \times 2.9 \mathrm{~cm}^{3}$ and the cardiac tissue domain size is $0.05 \times 2.9 \times 2.42 \mathrm{~cm}^{3}$. The computational domain of the composite geometry consists of 499,270 tetrahedral elements and 111,589 nodal points. The cardiac tissue domain comprises 266,846 tetrahedron and 59,292 nodal points. The computational domains, the control domains, $\Gamma_{1}$ and $\Gamma_{2}$, and the relevant subdomains are depicted in Figure 1.

The conductivity values were chosen to arrive at physiologically relevant conduction velocities of $0.64 \mathrm{~m} / \mathrm{s}$ and $0.41 \mathrm{~m} / \mathrm{s}$ along and transverse to the principal fiber axes, respectively, and to keep anisotropy ratios within the range of values reported in experimental studies [34]. A rule-based method was used to impose fiber orientations within the biventricular slice geometry [6] using fiber angles of -60॰ and 
+60 o at the endocardial and epicardial surfaces, respectively, and a smooth linear variation of fiber angles as a function of depth in between.
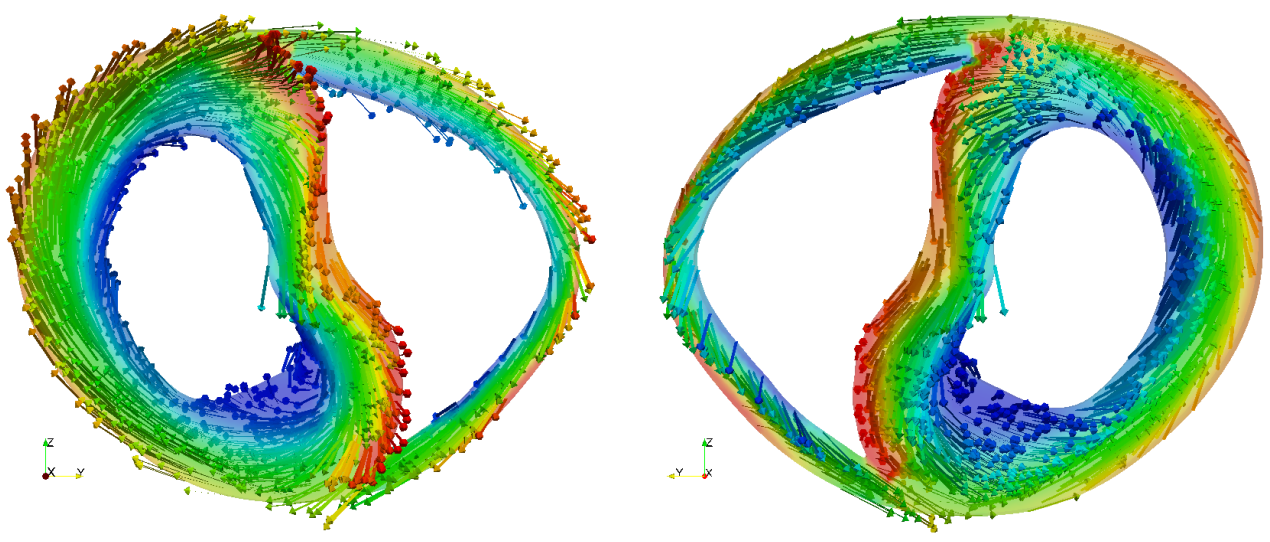

Figure 2: Fiber directions $a_{l}(x)$ in yz-plane at top and bottom of slab.

A standard $S 1-S 2$ stimulation protocol was applied to induce a reentrant activation pattern. First, an initial stimulus $S 1$ of $I_{t r}=30 \mu \mathrm{A} / \mathrm{cm}^{3}$ was applied at time $t=0.5 \mathrm{msec}$ for a duration of $5 \mathrm{msec}$ in the box $[-0.171,-0.121] \times[0.53,0.8] \times$ $[-1.52,-1]$ of the cardiac tissue sheet to initiate a planar wavefront. At time $t=$ $340 \mathrm{msec}$, when the critical recovery isoline arrived at point $(-0.185,0.61,-0.55)$, a second $S 2$ stimulus of strength $I_{t r}=30 \mu \mathrm{A} / \mathrm{cm}^{3}$ was applied in a small region of $0.4 \mathrm{~cm}$ radius for a duration of $5 \mathrm{msec}$. This $S 2$ stimulus generated a reentrant excitation wave front. The direct simulation was carried out until time $t=2500$ msec to ensure that the induced reentry is maintained for a prolonged period of time. The solution at $t=585 \mathrm{msec}$ was then chosen as the initial state for the optimal control experiment. The three temporal horizons: reentry induction, optimization duration, and post shock simulation as depicted in Figure 3.

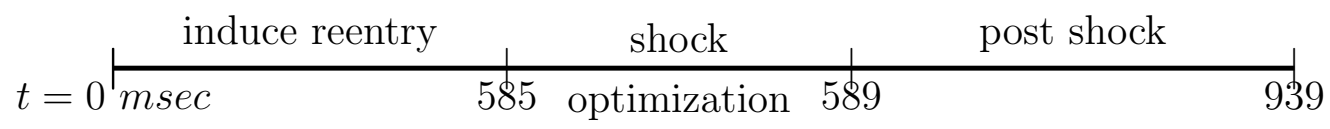

Figure 3: Different time horizons considered in the computations.

The initial solution of the extracellular potential, the transmembrane voltage and the gating variable for the optimization algorithm at time $t=585 \mathrm{msec}$ are depicted in Figure 4.

To fully specify the optimal control problem the desired trajectory of the transmembrane potential $\left(v_{d}\right)$ needs to be specified. Here it is chosen as a trajectory which corresponds to successful defibrillation. It is generated by solving once the primal problem with a stimulation $I_{e}(t)=30 \mathrm{~mA} / \mathrm{cm}^{3}$. Its graph looks similar to that which is shown in Figure 7 . The optimal control procedure then determines a stimulus which still leads to defibrillation but with less energy.

In our computations, the termination of the optimization algorithm is based on the following condition:

$$
\left\|\nabla J\left(\mathbf{I}_{e}^{k}\right)\right\|_{L^{2}} \leq 10^{-3} \cdot\left|J\left(\mathbf{I}_{e}^{k}\right)\right| \text { or }\left|J\left(\mathbf{I}_{e}^{k}\right)-J\left(\mathbf{I}_{e}^{k-1}\right)\right| \leq 10^{-4}
$$




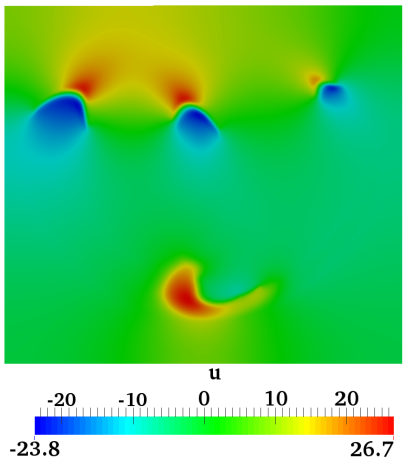

(a) extracellular potential

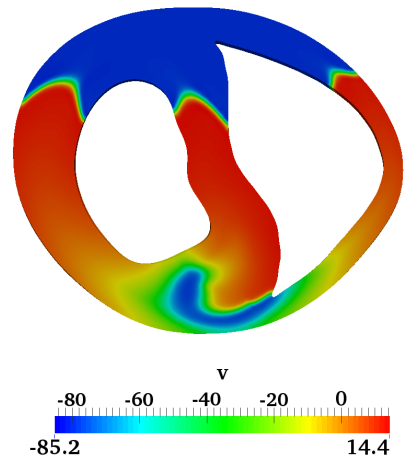

(b) transmembrane voltage

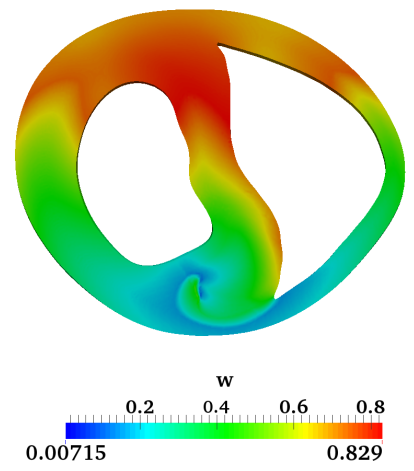

(c) gating variable

Figure 4: The solution $(u, v, w)$ at $t=585$ msec.

If this condition was not satisfied within a prescribed number of 12 iterations, the algorithm was terminated. If $\alpha_{2}$ is large enough stopping is achieved by (46). An Armijo type condition is imposed in the line search algorithm. The complete optimization code was developed based on the public domain FEM software package DUNE [4]. The presented numerical results were computed on a Linux cluster consisting of ten nodes where each node consists of 8 quad-core AMD Opteron processors 8356 clocked at $2.3 \mathrm{GHz}$ and equipped with 1TB RAM. All presented results are based on the parallel Newton-CG algorithms using 16 cores.

\section{Termination of reentry waves}

In the following we present the numerical results. This test case demonstrates that the optimal control strategy will compute the suitable optimal control for termination of reentry waves by properly choosing the weight of the control cost. Here the weight of the tracking term is fixed $\alpha_{1}=0.05$ and the weight of the control cost is investigated. Concerning the choice of the parameters $\alpha_{1}$ and $\alpha_{2}$ in Eq. (24), let us note that the optimal control only depends on the ratio $\frac{\alpha_{2}}{\alpha_{1}}$. In the optimization procedure $\alpha_{1}$ acts as a scaling of the adjoint variables $\left(p_{b}, p, q, r\right)$. For fixed $\alpha_{1}$, the weight $\alpha_{2}$ describes the relative weight of the cost of the control $I_{e}$. In our computation we set $\alpha_{1}=0.05$ and analyze the effect of varying $\alpha_{2}$.

\section{The desired trajectory using $I_{e}=30 \mathrm{~mA} / \mathrm{cm}^{3}$}

In this subsection, the desired trajectory is constructed using $I_{e}=30 \mathrm{~mA} / \mathrm{cm}^{3}$ by solving the primal system over the time period of $4 \mathrm{msec}$. Here we ensure that the computed solution at the shock period leads to successful defibrillation during the post shock simulations.

In this test case the initial value for the control is taken as $I_{e}=10 \mathrm{~mA} / \mathrm{cm}^{3}$. The norm of the gradient of the cost functional is shown in left hand side of Figure 5, where the norm of the gradient value is depicted on log scale for better reading at the last iterations of optimization algorithm. In this test case, the weight of the control cost is $\alpha_{2}=0.05,0.1,0.5,1.0$ and 5.0. We can observe that the norm of the gradient value is reduced during first iterations which is about 95.93. Then the reduction is very small at the last iterations and the smallest gradient value is 0.009 .

The optimal control value is depicted in Figure 6 for different weights of the 

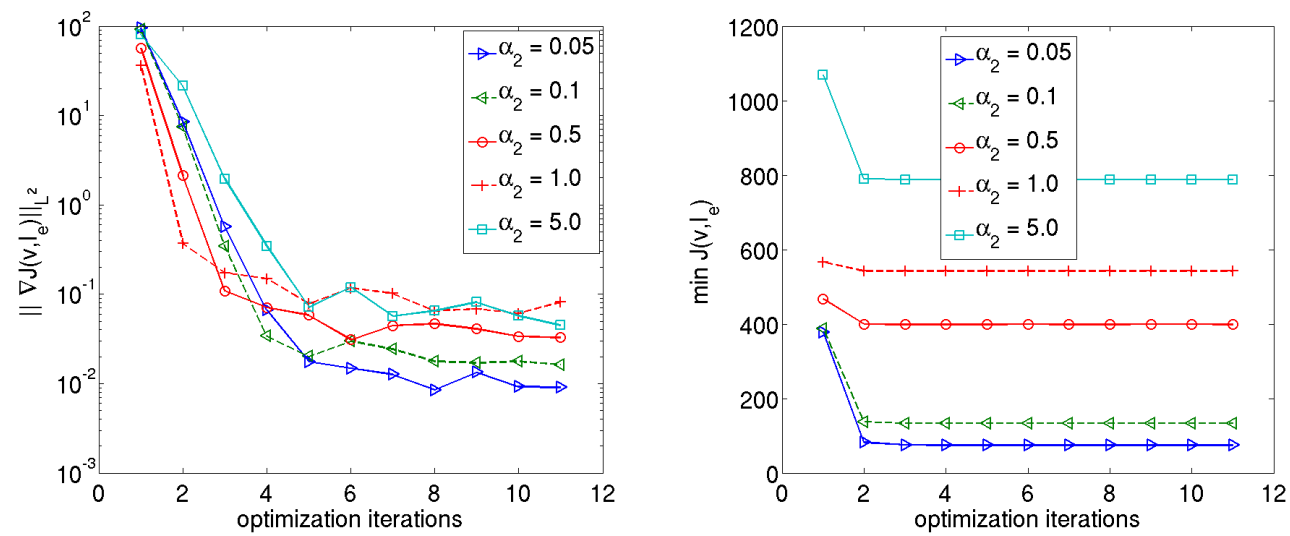

Figure 5: The gradient (log scale at $\mathrm{Y}$-axis) and the minimum value of the cost functional are shown on the left and right figures respectively.

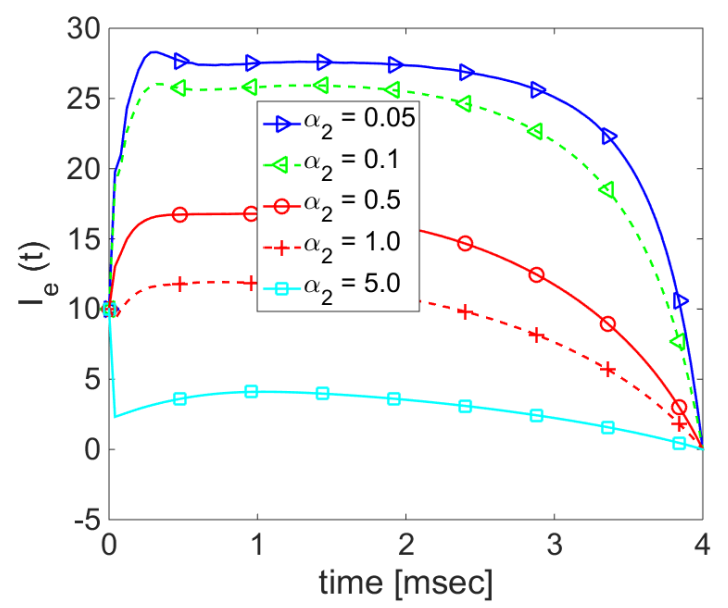

Figure 6: Optimal control.

costs. We observed the optimal control obtained for $\alpha_{2}=0.05,0.1$ and 0.5 leads to a successful defibrillation during the post shock simulations. For $\alpha_{2}=1$ and 5 the successful defibrillation was not achieved due to not sufficient field strength during the shock period. The obtained total current, $\int_{0}^{T}\left|I_{e}\right| \mathrm{d} t$, for $\alpha_{2}=0.05,0.1,0.5$, 1.0 and 5.0 is $98.6429,89.3688,53.7900,36.7685$ and $11.7117 \mathrm{~mA} / \mathrm{cm}^{3}$ respectively. From this experiment we observed that the minimum $\alpha_{2}=0.5$ is required to obtain a successful defibrillation. As a consequence we take this control trajectory value for our next test case. For this test case, the Newton optimization algorithm takes 11 outer iterations to achieve the desired tolerance and an average of 6 inner CG iterations were required to solve the Hessian system.

Remark 5.1. Let us make make a few comments on the performance of the optimization algorithm.

1)The stopping criteria plays an important role to obtain convergence to a local solution. We observed that the line search algorithm for the Newton's method rejects full step lengths at the beginning of the optimization algorithm and accepts full step length after about 10 iterations.

2) It is well known that the Newton method requires a good starting point to guar- 
antee its convergence. To address this issue for our situation, we constructed the initial guess by two optimization iterations of the nonlinear conjugate gradient method (NCG) with the Hager-Zhang update, see [30, page 121]. This ensured the convergence of the Newton's method in our computations. For example with the initial guess $I_{e}(t)=0$ the Newton-CG algorithm diverges, which with the initialization as just described it converges.

3) We observed that the state $v$ at time $560 \mathrm{msec}$ as initial state for the optimization did not lead to successful defibrillation in the direct numerical simulation. Moreover, increasing the strength of the external stimulus also did not lead a successful defibrillation. The initial state solution for the shock period at times 575, 580 and 590 msecs led to a successful termination of reentry waves during the post shock simulations. This is consistent with the fact that the time of shock is essential for the successful defibrillation.


Figure 7: The optimal state solution of transmembrane voltage $v$ during the shock period at times $t=585.32,587.4$ and $589 \mathrm{msec}$.

The 3D colored plots of transmembrane voltage solution (color bar is re-scaled for better comparison with the uncontrolled solution) during the shock period is illustrated in Figure 7 for different time instances and for $\alpha_{2}=0.5$. As can be seen in the first panel of Fig. 7 at $t=585.32 \mathrm{~ms}$, a large number of virtual electrodes appear within the cardiac tissue domain. During the post shock simulation, these small-scale polarizations start to diffuse out at time $t=589.52 \mathrm{~ms}$, see Figure 8 . Then the appearance of numerous small-scale virtual electrodes all over the tissue in both excitable gap as well as in depolarized regions effectively block the further propagation of the spiral wave. At time $941 \mathrm{msec}$ the reentrant wave disappears completely from the cardiac tissue domain.
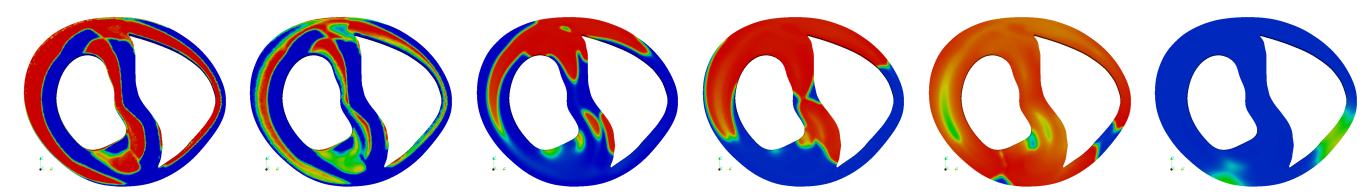

Figure 8: The solution of transmembrane voltage $v$ during the post-shock period at times $t=589.13,589.52,607.31,630.41,723.06$ and $922.44 \mathrm{msec}$ (color bar should be the same as in Figure 7).

Remark 5.2. From the above results we conclude that the optimal control strategy is successful in the sense that it can provide a control that leads to successful defibrillation at a lower cost than controls based on educated guesses. One can think of iterating the chosen procedure and replacing in a second experiment the desired trajectory $v_{d}$ by the trajectory corresponding to the optimal control $I_{e}$ for the choice 
$\alpha_{2}=0.5$, which is still defibrillating. We carried out this experiment and observed that no significant further reduction of the energy required for defibrillation can be achieved.

\section{The desired trajectory using $I_{e}=17 \mathrm{~mA} / \mathrm{cm}^{3}$}

In this test case, we use the constant $I_{e}=17 \mathrm{~mA} / \mathrm{cm}^{3}$ for creating the desired trajectory which is just sufficient, the maximum value in that curve, to terminate the reentry wave by the knowledge from previous test case. This test case is considered to demonstrate the robustness of optimal control techniques. In this test cases, different regularization parameter values are used to bring the computed solution as close as possible to the desired trajectory so that it will lead to terminate the reentrant wave during the post shock solution. The norm of the gradient value at the left and the computed optimal control value over the time at the right of Figure 9 is shown for different regularization parameter values. Here, from observed post shock simulations, we observe that $\alpha_{2}=0.02$ is sufficient to fulfill the desired objective which is to terminate the reentrant wave during the post shock solution. The cases $\alpha_{2}=0.08$ and 0.8 did not lead to a successful defibrillation. The total current for the construction of the desired trajectory is $68 \mathrm{~mA} / \mathrm{cm}^{3}$, whereas the optimal control approach requires only $53.4475 \mathrm{~mA} / \mathrm{cm}^{3}$. Thus we can further decrease but this decrease is significantly smaller than the case described in the previous subsection. The optimal control for this test case is different from the previous one, but the total current is approximately same. A comparison between two optimal control solutions is depicted in Figure 10.
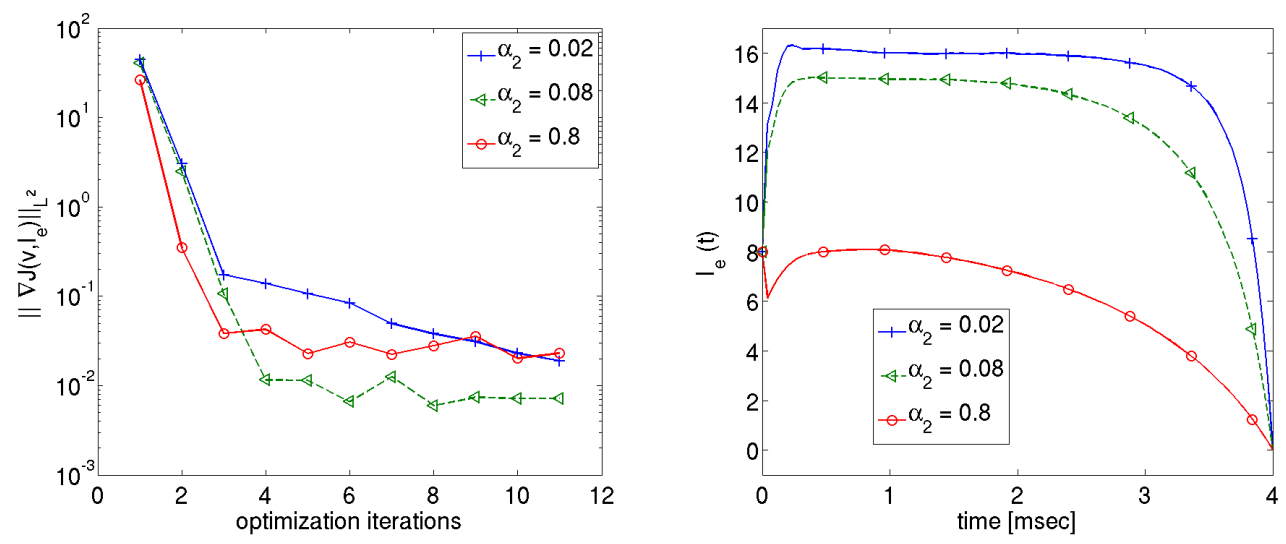

Figure 9: The gradient value (log scale at Y-axis) of the cost functional and the optimal control value are shown on the left and right figures respectively.

\section{Parallel efficiency}

In general, solving the primal or adjoint equations dominates overall computational cost in an optimal control solver. Due to the solving of state equations on two different geometries $\Omega$ and $\Omega_{H}$, it plays a important role to achieve a strong scalability over the solution of complete direct problem. In this study, the benchmark simulations were performed on the tissue domain surrounded by a bath domains shown in Figure 1 to assess the strong scaling properties of the complete bidomain system solvers used in this study. The test case 2 was adopted and the weight of the control 


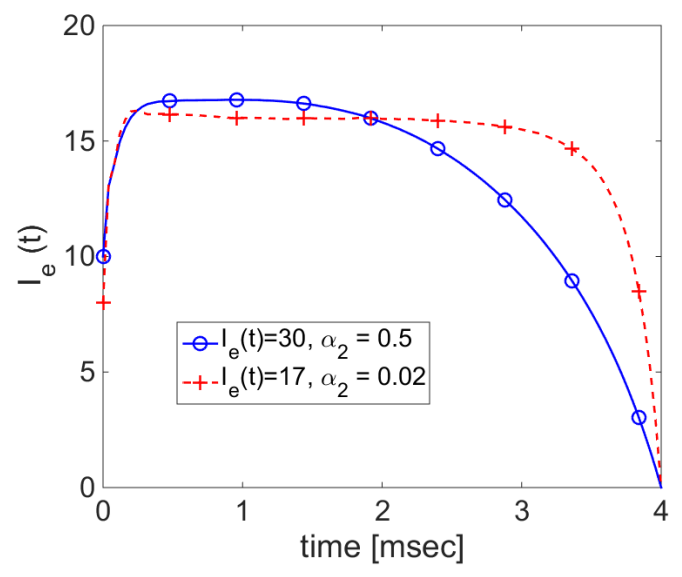

Figure 10: Comparison between two optimal control solutions.

cost is $\alpha_{2}=0.02$. The parallel efficiency of this benchmark was demonstrated for different components of the solver as well as for the complete optimization. The strong scalability was tested up to 16 cores and the parallel efficiency was computed as

$$
e=\frac{1}{N} \frac{T_{1}}{T_{N}}
$$

where $T_{1}$ and $T_{N}$ are total CPU time of a reference simulation and $T_{N}$ is the total CPU time on $N$ number of cores, respectively.

Here we compare the computing times of reference computation on single CPU and the parallel efficiency up to 16 CPUs. First we give the component-wise CPU times for the primal solver at first iteration of optimization solver. The elliptic solver took 414.98 seconds and the coupled PDE/ODE solver consumed 1091.21 seconds. The first primal solve took 1511.81 seconds and similarly the corresponding dual solve took 1547.20 seconds. The complete optimization algorithm has taken 6.23 days on one CPU. For the parallel efficiency the data is shown in Figure 11.

Now we turn to the communication CPU times between the integrated and tissue geometries. We observed that the communication time for sending the elliptic solution from the whole domain to the tissue domain attained 0.152 seconds and 0.612 seconds for communicating the transmembrane solution from the tissue domain to the whole domain on one CPU. On 16 CPUs the CPU times for communicating the data are 0.316 and 0.332 respectively. The CPU time for building the look up table for communicating the data between the two grids took 0.9 seconds on 16 CPUs. We can observe that the communication CPU times between the two geometries is almost negligible as compared to the solution of the PDE system. Here we point out that, the load balancing of the cardiac tissue grid on 16 cores assigns approximately 4,000 nodal points on each core. The drop in the parallel efficiency can be attributed to the unfavorable surface-to-volume ratio of local domains. With increasing number of cores, the relation between the local compute work performed on inner nodes of the domain and the data communication which are proportional to the size of shared domain interfaces, deteriorates, thus impeding further efficient scaling. 


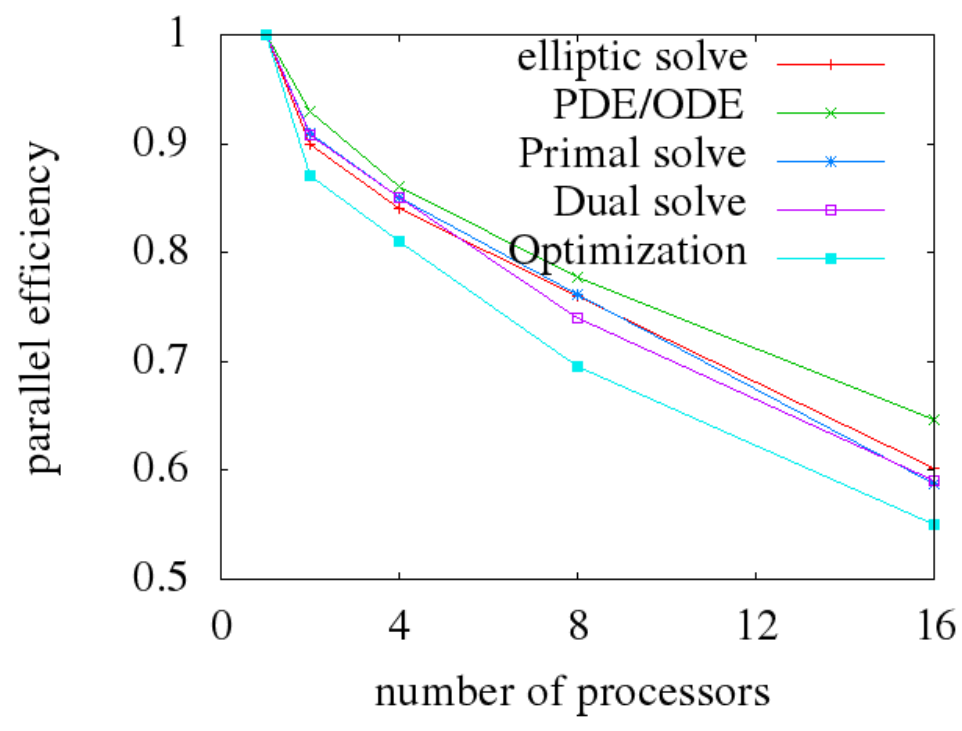

Figure 11: Parallel efficiency over the numbers of processors

\section{Summary}

It was demonstrated that optimal control techniques allow successful defibrillation with less energy when compared to ad-hoc choices of the shock strength. Compared to our earlier work the electrophysiology modeling was significantly expanded by including a bath domain as well as imaging based conductivity tensors which represent more realistically the orthotropic architecture of the cardiac tissue structure. Moreover, we adopted the simplified Mitchell-Schaeffer ionic model which describes physiological properties such as activation dynamics, inward and outward currents and the concomitant action potential shape in more detail than the ionic models used in previous optimal control studies. Due to these changes we observed that the fibrillatory activity and the appearance of virtual electrode polarization during the shock period is different from our previous optimal control studies $[16,14]$ and relates more close to other DNS studies [21].

We should also mention that with a bath domain included and with the structurally more realistic orthotropic tissue properties, the FitzHugh-Nagumo ionic model did not allow successful defibrillation in our computations on the 3D slice geometry while the Mitchell-Schaeffer did. The numerical results demonstrate that we have found a promising approach for an optimal control treatment of defibrillation problems, the question of appropriate locations of applying control and the applying stimulus timings of optimal control strategies for such problems remains a most challenging one.

We have encountered that the initial guess of the control and the stopping criteria plays a crucial role for convergence of the optimization algorithm. The optimization algorithm was performed well for the given desired trajectory, which is obtained from the computed optimal control, to achieve the desired objective and demonstrated that no significant further reduction of the energy is required.

Solving the optimal control problem of bidomain equations in a whole heart model would be an extremely costly endeavor. However, the forward simulation benchmark data strongly support the notion, see [28] for benchmark results of the forward problem of a rabbit ventricular whole heart model, that such simulations 
are certainly feasible in future. Replacing the simplified phenomenological models by various biophysically detailed models is our primary goal in the near future which makes difference in after the break of the shock, depending on how refractoriness and recovery from refractoriness.

\section{Acknowledgment}

The authors gratefully acknowledge the Austrian Science Foundation (FWF) for financial support under SFB 032, "Mathematical Optimization and Applications in Biomedical Sciences" and the Austrian Academy of Sciences (OÄW).

\section{References}

[1] CellML Model Repository. http://models.cellml.org/cellml.

[2] A comparison of antiarrhythmic-drug therapy with implantable defibrillators in patients resuscitated from near-fatal ventricular arrhythmias. the antiarrhythmics versus implantable defibrillators (avid) investigators. $N$ Engl J Med, 337(22):1576-1583, Nov 1997.

[3] G. H. Bardy, B. Hofer, G. Johnson, P. J. Kudenchuk, J. E. Poole, G. L. Dolack, M. Gleva, R. Mitchell, and D. Kelso. Implantable transvenous cardioverterdefibrillators. Circulation, 87(4):1152-68, 1993.

[4] P. Bastian, M. Blatt, A. Dedner, C. Engwer, R. Klöfkorn, R. Kornhuber, M. Ohlberger, and O. Sander. A generic grid interface for parallel and adaptive scientific computing. Part II: implementation and tests in DUNE. Computing, 82(2):121-138, July 2008.

[5] P. Bastian, F. Heimann, and S. Marnach. Generic implementation of finite element methods in the distributed and unified numerics environment (DUNE). Kybernetika, 46(2):294-315, 2010.

[6] J. D. Bayer, R. C. Blake, G. Plank, and N. A. Trayanova. A novel rule-based algorithm for assigning myocardial fiber orientation to computational heart models. Ann Biomed Eng, 40(10):2243-2254, Oct 2012.

[7] M. J. Bishop and G. Plank. Representing cardiac bidomain bath-loading effects by an augmented monodomain approach: application to complex ventricular models. IEEE Trans Biomed Eng, 58(4):1066-1075, Apr 2011.

[8] M. J. Bishop, E. Vigmond, and G. Plank. Cardiac bidomain bath-loading effects during arrhythmias: interaction with anatomical heterogeneity. Biophys $J$, 101(12):2871-2881, Dec 2011.

[9] M. Blatt. A parallel algebraic multigrid method for elliptic problems with highly discontinuous coefficients. PhD thesis, Ruprechts-Karls-Universität Heidelberg, 2010 .

[10] P. Bochev and R. B. Lehoucq. On the finite element solution of the pure neumann problem. SIAM Rev., 47:50-66, January 2005. 
[11] G. Boriani, M. Biffi, P. Silvestri, C. Martignani, C. Valzania, I. Diemberger, C. Moulder, G. Mouchawar, M. Kroll, and A. Branzi. Mechanisms of pain associated with internal defibrillation shocks: results of a randomized study of shock waveform. Heart Rhythm, 2(7):708-713, Jul 2005.

[12] M. Boulakia, M. Fernandez, J.-F. Gerbeau, and N. Zemzemi. A coupled system of PDEs and ODEs arising in electrocariograms modelling. Applied Mathematics Research eXpress, 2009.

[13] N. Chamakuri and K. Kunisch. Higher order optimization and adaptive numerical solution for optimal control of monodomain equations in cardiac electrophysiology. Appl. Numer. Math., 61:53-65, 2011.

[14] N. Chamakuri, K. Kunisch, and G. Plank. Optimal control approach to termination of re-entry waves in cardiac electrophysiology. Journal of Mathematical Biology, 67(2):359-388.

[15] N. Chamakuri, K. Kunisch, and G. Plank. Numerical solution for optimal control of the reaction-diffusion equations in cardiac electrophysiology. Computational Optimization and Applications, 49:149-178, 2011. 10.1007/s10589009-9280-3.

[16] N. Chamakuri, K. Kunisch, and G. Plank. On boundary stimulation and optimal boundary control of the bidomain equations. Mathematical Biosciences, 245(2):206 - 215, 2013.

[17] K. De Bruin and W. Krassowska. Electroporation and shock-induced transmembrane potential in a cardiac fiber during defibrillation strength shocks. Ann Biomed Eng, 26:584-596, 1998.

[18] A. Dedner, C. Rohde, B. Schupp, and M. Wesenberg. A parallel, load-balanced mhd code on locally-adapted, unstructured grids in 3d. Computing and Visualization in Science, 7(2):79-96, 2004.

[19] F. H. Fenton, S. Luther, E. M. Cherry, N. F. Otani, V. Krinsky, A. Pumir, E. Bodenschatz, and R. F. Gilmour, Jr. Termination of atrial fibrillation using pulsed low-energy far-field stimulation. Circulation, 120(6):467-476, Aug 2009.

[20] M. G. Fishler. Theoretical predictions of the optimal monophasic and biphasic defibrillation waveshapes. IEEE Trans Biomed Eng, 47(1):59-67, Jan 2000.

[21] M. G. Hillebrenner, J. C. Eason, and N. A. Trayanova. Mechanistic inquiry into decrease in probability of defibrillation success with increase in complexity of preshock reentrant activity. American Journal of Physiology - Heart and Circulatory Physiology, 286(3):H909-H917, 2004.

[22] J. Huang, G. P. Walcott, R. B. Ruse, S. J. Bohanan, C. R. Killingsworth, and R. E. Ideker. Ascending-ramp biphasic waveform has a lower defibrillation threshold and releases less troponin i than a truncated exponential biphasic waveform. Circulation, 126(11):1328-1333, Sep 2012.

[23] G. Karypis and V. Kumar. A fast and high quality multilevel scheme for partitioning irregular graphs. SIAM Journal on Scientific Computing, 20(1):359392, 1998. 
[24] J. P. Keener. Modeling electrical activity of cardiac cells, two variable models. www.math.utah.edu/ keener/lectures/ionic_models/Two_variable_ models.

[25] J. Lang. Adaptive Multilevel Solution of Nonlinear Parabolic PDE Systems, volume 16 of Lecture Notes in Computational Science and Engineering. SpringerVerlag, Berlin, 2001.

[26] S. Luther, F. H. Fenton, B. G. Kornreich, A. Squires, P. Bittihn, D. Hornung, M. Zabel, J. Flanders, A. Gladuli, L. Campoy, E. M. Cherry, G. Luther, G. Hasenfuss, V. I. Krinsky, A. Pumir, R. F. Gilmour, Jr, and E. Bodenschatz. Low-energy control of electrical turbulence in the heart. Nature, 475(7355):235239, Jul 2011.

[27] C. Mitchell and D. Schaeffer. A two-current model for the dynamics of cardiac membrane. Bulletin of Mathematical Biology, 65(5):767-793, 2003.

[28] A. Neic, M. Liebmann, E. Hoetzl, L. Mitchell, E. J. Vigmond, G. Haase, and G. Plank. Accelerating cardiac bidomain simulations using graphics processing units. IEEE Trans. Biomed. Engineering, 59(8):2281-2290, 2012.

[29] S. Niederer, L. Mitchell, N. Smith, and G. Plank. Simulating human cardiac electrophysiology on clinical time-scales. Front Physiol, 2:14, 2011.

[30] J. Nocedal and S. J. Wright. Numerical Optimization. Springer Verlag, New York, second edition edition, 2006.

[31] L. F. Pavarino and S. Scacchi. Multilevel additive schwarz preconditioners for the bidomain reaction-diffusion system. SIAM J. Sci. Comput., 31:420-443, October 2008.

[32] G. Plank, M. Liebmann, R. W. dos Santos, E. Vigmond, and G. Haase. Algebraic multigrid preconditioner for the cardiac bidomain model. IEEE Trans Biomed Eng., 54(4):585-596, 2007.

[33] D. Rohmer, A. Sitek, and G. T. Gullberg. Reconstruction and visualization of fiber and laminar structure in the normal human heart from ex vivo diffusion tensor magnetic resonance imaging (dtmri) data. Invest Radiol, 42(11):777-789, Nov 2007.

[34] B. J. Roth. Electrical conductivity values used with the bidomain model of cardiac tissue. IEEE Trans Biomed Eng, 44(4):326-328, Apr 1997.

[35] A. T. Sambelashvili, V. P. Nikolski, and I. R. Efimov. Virtual electrode theory explains pacing threshold increase caused by cardiac tissue damage. Am J Physiol Heart Circ Physiol, 286(6):H2183-H2194, Jun 2004.

[36] J. C. Schuder, H. Stoeckle, J. A. West, and P. Y. Keskar. Transthoracic ventricular defibrillation in the dog with truncated and untruncated exponential stimuli. IEEE Trans Biomed Eng, 18(6):410-415, Nov 1971.

[37] N. G. Sepulveda, B. J. Roth, and J. P. Wikswo, Jr. Current injection into a two-dimensional anisotropic bidomain. Biophys J, 55(5):987-99, 1989. 
[38] E. Sobie, R. Susil, and L. Tung. A generalized activating function for predicting virtual electrodes in cardiac tissue. Biophys J, 73(3):1410-23, 1997.

[39] L. Tung. A bi-domain model for describing ischemic myocardial DC potentials. PhD thesis, MIT, Cambridge, MA, 1978.

[40] H. A. van der Vorst. Bi-CGSTAB: A fast and smoothly converging variant of bicg for the solution of nonsymmetric linear systems. SIAM J. Sci. Stat. Comput., 13:631-644, 1994.

[41] F. J. Vetter and A. D. McCulloch. Three-dimensional analysis of regional cardiac function: a model of rabbit ventricular anatomy. Prog Biophys Mol Biol, 69(2-3):157-183, 1998.

[42] E. Vigmond, F. Vadakkumpadan, V. Gurev, H. Arevalo, M. Deo, G. Plank, and N. Trayanova. Towards predictive modelling of the electrophysiology of the heart. Exp Physiol, 94(5):563-577, May 2009.

[43] G. P. Walcott, R. G. Walker, A. W. Cates, W. Krassowska, W. M. Smith, and R. E. Ideker. Choosing the optimal monophasic and biphasic waveforms for ventricular defibrillation. J Cardiovasc Electrophysiol, 6(9):737-750, Sep 1995. 